\title{
Efeito de diferentes métodos de indução à puberdade sobre a resposta reprodutiva em novilhas nelore
}

\author{
Lucas Henrique Ribeiro MAGI ${ }^{1}$, Isabela Lara DAMIÃO ${ }^{1}$, Mylla Cristina Freitas MORAIS'1, \\ Richarlla Aparecida Buscariol SILVA¹, Sofia Regina POLIZELLE ${ }^{1}$, \\ Danila Fernanda Rodrigues FRIAS ${ }^{*}$
}

\author{
${ }^{1}$ Universidade Brasil, Fernandopólis, SP, Brasil. \\ (Orcid: 0000-0003-2958-2983; 0000-0002-3662-120X; 0000-0001-7780-1269; 0000-0002-3190-8332; 0000-0003-1955-3951; *) \\ *E-mail: danila.frias@universidadebrasil.edu.br (Orcid: 0000-0001-8621-3338)
}

Recebido em 07/08/2020; Aceito em 28/09/2020; Publicado em 27/10/2020.

\begin{abstract}
RESUMO: Analisou-se a influência de diferentes protocolos de indução de puberdade em novilhas Nelore por meio da avaliação de dados referentes a 1528 animais relacionados ao resultado da avaliação ginecológica antes da realização do protocolo de indução, escore de condição corporal (ECC), peso médio e idade, protocolo de indução utilizado e resultado do diagnóstico de gestação. Após a tabulação dos dados foram realizadas análises estatísticas por meio do teste de Qui-Quadrado. Com relação ao ECC, $100 \%$ das fêmeas foram classificadas entre 3 e 3,5. Quanto a idade e peso, 30,9\% possuíam em média 14 meses e pesavam cerca de 270 $\mathrm{kg}$, e $69,1 \%$ cerca de 20 meses com peso médio de $320 \mathrm{~kg}$. As novilhas que passaram por protocolo de indução apresentaram 8,3\% a mais na taxa de prenhez. O protocolo que utilizou apenas progesterona injetável (P4) de longa ação apresentou os mesmos resultados do tratamento com dispositivo intravaginal reutilizável associado ao cipionato de estradiol. Conclui-se que a progesterona é eficaz para indução de puberdade em novilhas a partir dos 14 meses de idade com peso médio de $270 \mathrm{~kg}$, além disso, a utilização da P4 injetável pode ser uma alternativa eficaz para uso como protocolo de indução de novilhas pré-puberes.
\end{abstract}

Palavras-chave: indução de ovulação; progesterona; taxa de concepção; tratamento hormonal.

\section{Effect of different puberty-induction methods on reproductive response in nellore heifers}

\begin{abstract}
Examines the influence of different puberty induction protocols in Nellore heifers. Data referring to 1528 Nellore heifers related to the result of gynecological evaluation before the induction protocol; body condition score (BCS); average heifer weight and age; induction protocol used; and result of pregnancy diagnosis. After data tabulation, statistical analyses were performed using the Chi-Square test. For BCS, 100\% of the females were classified between 3 and 3.5. In terms of age and weight, 30.9\% had an average age of 14 months and weighed approximately $270 \mathrm{~kg}$, whereas $69.1 \%$ were around 20 months old, with an average weight of $320 \mathrm{~kg}$. Heifers that underwent an induction protocol showed an $8.3 \%$ higher pregnancy rate. The protocol that involved only long-acting injectable progesterone (P4) provided the same results as the treatment with the reusable intravaginal device associated with estradiol cypionate. In conclusion, progesterone is effective for inducing puberty in Nellore heifers from 14 months of age with an average weight of $270 \mathrm{~kg}$. in addition, the use of injectable P4 can be an effective alternative as an induction protocol for prepubertal Nellore heifers.

Keywords: conception rate; hormonal treatment; ovulation induction; progesterone.
\end{abstract}

\section{INTRODUÇÃO}

O Brasil possui o maior rebanho comercial bovino do mundo, composto por cerca de 214 milhões de cabeças, e destas, $80 \%$ são animais de raças zebuínas (Bos taurus indicus). A raça Nelore compreende cerca de $90 \%$ do rebanho zebuíno nacional, e este fato está relacionado a sua grande adaptabilidade ao ambiente tropical nacional e a alta produtividade (IBGE, 2018; ARAUJO et al., 2018).

Mesmo a raça Nelore sendo tão difundida no Brasil, as fêmeas são consideradas tardias sexualmente, pois enquanto animais taurinos atingem a puberdade com cerca de 10 a 15 meses, os zebuínos chegam por volta dos 16 a 40 meses (HAFEZ; HAFEZ, 2004). Desta forma, existe a busca constante de estratégias efetivas que melhorem a eficiência reprodutiva das fêmeas Nelore, somado a qualidade de seu produto final (ARAUJO et al., 2018).

A puberdade ocorre quando a fêmea apresenta o primeiro estro com ovulação viável e crescimento de corpo lúteo funcional, além de manifestar todos os eventos de comportamento de cio (PERRY, 2016; SOUZA et al., 2018; ARAUJO et al., 2019).

A idade a puberdade é um fator muito importante na eficiência reprodutiva, produtiva e econômica de um sistema de produção de bovinos, pois a prenhez precoce reduz o período de recria, evita uso de pastagens por novilhas com idade avançada e improdutivas, aumenta o número de nascimentos na propriedade e, quanto mais cedo esta fêmea se reproduzir, maior será sua vida produtiva na fazenda 
(CARDOSO et al., 2007; ELER et al., 2010; FERREIRA et al, 2012; ARAUJO, et al., 2018).

Vários fatores estão relacionados com a indução da puberdade em novilhas, como a genética, nutrição, ambiente, e presença de machos (ARAUJO, et al., 2019). O peso (cerca de 50 a $57 \%$ do peso adulto) e o desenvolvimento corporal são fatores muito importantes e auxiliam na identificação de animais potencialmente aptos para iniciar a atividade reprodutiva (MARSON; GUIMARAES; MIRANDA NETO, 2004; ENDECOTT et al., 2013).

A genética também influencia pois, animais Bos taurus taurus tem maior precocidade sexual do que os Bos taurus indicus, que são tardios, e possuem idade média ao primeiro parto aos 3 ou 4 anos de idade nos rebanhos brasileiros (DAY; NOGUEIRA, 2013; MALHADO et al., 2013).

Devido a estes fatores, as novilhas podem demorar a atingir a puberdade, acarretando em atraso na concepção (VASCONCELOS et al., 2017). Quando se deseja utilizar estas fêmeas na estação reprodutiva, é necessário que a mesma atinja a puberdade antes, pois ela é considerada realmente fértil a partir do terceiro cio, desta forma é desejável que a novilha entre em puberdade aos 13 meses, para conceber aos 15 e apresentar o primeiro parto aos 24 meses (FIGUEIREDO et al., 2008; SOUZA et al., 2018).

Como já relatado, animais zebuínos são tardios sexualmente, por isso, as novilhas dificilmente atingem a maturidade sexual aos 15 meses de forma espontânea, necessitando para isso a utilização de métodos de indução da puberdade (DAY; NOGUEIRA, 2013).

A terapia hormonal é a técnica mais utilizada para indução de ovulação em novilhas, pois utiliza-se hormônios, que modulam o funcionamento do eixo hipotálamo-hipofisário, aumentando a secreção de gonadotrofinas e induzindo a puberdade (BÓ et al., 2018).

Nos últimos anos vários protocolos hormonais têm sido utilizados para induzir a puberdade em novilhas. Estes protocolos se baseiam na associação ou não de vários hormônios, destacando-se o uso dos dispositivos de liberação de progesterona associado ao uso de estradiol para sincronizar a emergência da onda folicular (BÓ et al., 2018; SANTOS et al., 2018b).

Neste contexto, hipotetizamos que o uso de protocolos para induzir a puberdade em novilhas com uso de progesterona injetável (P4) poderá ser uma alternativa ao produtor. Assim, objetivou-se analisar a influência de diferentes protocolos de indução de puberdade em novilhas da raça Nelore.

\section{MATERIAL E MÉTODOS}

Para este estudo, foram avaliados dados referentes a 1581 novilhas Nelore provenientes de rebanhos localizados nos estados de Tocantins e Bahia, criadas em sistema extensivo, sob o mesmo manejo nutricional e sanitário, durante a estação reprodutiva 2018/2019 e 2019/2020.

Os dados analisados foram referentes ao resultado da avaliação ginecológica antes da realização do protocolo de indução, escore de condição corporal (ECC), peso médio e idade das novilhas, protocolo de indução utilizado e resultado do diagnóstico de gestação para a primeira IATF (Inseminação artificial em tempo fixo).

O ECC foi obtido pela avaliação visual utilizando escala de 1 a 5 com intervalo de 0,5 , onde 1 representava a fêmea com pouca massa muscular e cobertura de gordura e 5 a fêmea com excesso dessas duas características (JAUME; MORAES, 2002).

Para seleção das fêmeas pré-púberes foi realizado palpação retal e ultrassonografia, das quais foram retiradas 53 fêmeas púberes do banco de dados. Dos dados das fêmeas pré-púberes que foram analisados, 898 fêmeas provenientes da estação 2018/2019 e 630 fêmeas da estação 2019/2020. Estes animais apresentavam ECC entre 3 e 3,5, e peso de $270 \pm 9$ as com 14 meses, e $320 \pm 11$ as com 20 meses de idade.

Os animais avaliados foram separados em 3 grupos, de acordo com o protocolo de indução utilizado, e um o grupo controle, ou seja, novilhas que não receberam estímulo hormonal. Os grupos, de acordo com os protocolos foram:

Grupo 1: Protocolo para indução com progesterona (P4) injetável via intramuscular profunda.

D0 - $1 \mathrm{~mL}(150 \mathrm{mg})$ de P4 injetável;

D24 - Início do protocolo para de IATF.

Grupo 2: Protocolo para indução com implante de progesterona reutilizável.

D0 - colocar implante de progesterona ( $4^{\circ}$ uso);

D12 - aplicar $0,3 \mathrm{~mL}$ de cipionato de estradiol e retirar o implante;

D24- início do protocolo de IATF.

Grupo 3: Novilhas que não receberam protocolo de indução.

Após a realização do protocolo de indução, todas as fêmeas passaram por manejos de protocolos de IATF conforme descrito:

D0: inserção do implante de progesterona $+2,0 \mathrm{~mL}$ de benzoato de estradiol;

D7: 1,5mL de prostaglandina;

D9: Retirada do implante de progesterona $+0,3 \mathrm{~mL}$ cipionato de estradiol $+1,5 \mathrm{~mL}$ de gonadotrofina coriônica equina (eCG);

D11: Inseminação Artificial.

A quantidade de novilhas por grupo está descrita na Tabela 1.

Tabela 1. Novilhas induzidas separadas de acordo com os protocolos de indução utilizados e grupo controle.

Table 1. Induced heifers separated according to the used induction protocols and control group.

\begin{tabular}{cccc}
\hline $\begin{array}{c}\text { Estação } \\
\text { Reprodutiva }\end{array}$ & Grupo 1 & Grupo 2 & Grupo 3 \\
\hline $2018 / 2019$ & 381 & 293 & 224 \\
$2019 / 2020$ & 153 & 246 & 231 \\
\hline Total & 534 & 539 & 455 \\
\hline *Grupo 1: P4 injetável; Grupo 2: implante de progesterona + cipionato de
\end{tabular}

*Grupo 1: P4 injetável; Grupo 2: implante de progesterona + cipionato de estradiol; Grupo 3: não receberam protocolo de indução.

Após 24 dias do protocolo de indução, as novilhas foram expostas ao protocolo de IATF e inseminadas. O sêmen utilizado foi de touros da raça Nelore, e antes da inseminação uma dose de cada partida foi descongelada e realizada a avalição de qualidade e viabilidade do material. Em seguida a inseminação foi realizada pelo médico veterinário responsável pelo rebanho. 
O diagnóstico de gestação foi realizado 40 dias pós inseminação com uso de equipamento de ultrassom. Os animais foram considerados como prenhes quando uma vesícula embrionária com embrião com presença de batimentos cardíacos foi observada.

Após a tabulação dos dados coletados neste trabalho, foram realizadas análises estatísticas por meio do teste de Qui-Quadrado utilizando-se o software R versão 3.6.3.

\section{RESULTADOS}

No primeiro dia do protocolo de pré-indução, as novilhas foram classificadas de acordo com o ECC, seguindo escala de 1 a 5. Das 898 fêmeas da estação 2018/2019, 61\% foram classificadas com ECC 3, e 49\% ECC 3,5, e as novilhas da estação 2019/2020, 100\% estavam com ECC 3,5\%.

Foi observado que $30,9 \%$ das novilhas nelore encontravam com média de 14 meses de idade e pesando 270 $\mathrm{kg}$ e $69,1 \%$ das novilhas com cerca de 20 meses de idade e com peso médio de $320 \mathrm{~kg}$ (Tabela 2).

Tabela 2. Peso e idade de novilhas Nelore avaliadas nas estações reprodutivas 2018/2019 e 2019/2020.

Table 2. Weight and age of Nellore heifers evaluated in breeding seasons 2018/2019 and 2019/2020.

\begin{tabular}{ccccc}
\hline \multirow{2}{*}{$\begin{array}{c}\text { Estação } \\
\text { reprodutiva }\end{array}$} & \multicolumn{2}{c}{ Peso médio } & \multicolumn{2}{c}{ Idade média } \\
\hline $2018 / 2019$ & 231 & $620 \mathrm{~kg}$ & 14 meses & 20 meses \\
\hline $2019 / 2020$ & 241 & 389 & 231 & 667 \\
\hline $\begin{array}{c}\text { Total } \\
\text { Animais }\end{array}$ & 472 & 1056 & 241 & 389 \\
\hline
\end{tabular}

Os resultados referentes a taxa de prenhez relacionada ao uso ou não de protocolo de indução estão expressos na Tabela 3.

Tabela 3. Resultado do diagnóstico de gestação de acordo com o uso ou não de protocolo de indução em novilhas Nelore.

Table 3. Result of pregnancy diagnosis according to the use or not of induction protocol in Nellore heifers.

\begin{tabular}{cccc}
\hline $\begin{array}{c}\text { Estação } \\
\text { Reprodutiva }\end{array}$ & $\begin{array}{c}\text { Grupo 1 } \\
\text { Índice de } \\
\text { Prenhez }\end{array}$ & $\begin{array}{c}\text { Grupo 2 } \\
\text { Índice de } \\
\text { Prenhez }\end{array}$ & $\begin{array}{c}\text { Grupo 3 } \\
\text { Índice de } \\
\text { Prenhez }\end{array}$ \\
\hline $2018 / 2019$ & $53,5 \%$ & $53 \%$ & $45 \%$ \\
$2019 / 2020$ & $54,5 \%$ & $54 \%$ & $46 \%$ \\
\hline Média & $54 \%$ & $53,5 \%$ & $45,5 \%$ \\
\hline
\end{tabular}

*Grupo 1: P4 injetável; Grupo 2: implante de progesterona + cipionato de estradiol; Grupo 3: não receberam protocolo de indução.

O grupo de novilhas que passaram por protocolo de indução apresentaram melhor taxa de prenhez, com média de $8,3 \%$ de incremento nos resultados.

Comparando-se o resultado do diagnóstico de gestação com relação ao uso dos diferentes protocolos e relacionando ao grupo não induzido, os resultados estão descritos na Tabela 4.

Os grupos de animais induzidos apresentaram melhores taxa de prenhez $(P<0,05)$ quando comparados ao grupo 3 (sem protocolo de indução) (Tabela 5)

Ao avaliar a idade das novilhas pré-puberes e relacionar com o protocolo de indução utilizado, não se encontrou diferença entre as idades (Grupo $1 P=0,2187$, Grupo 2 $P=0,1534$ ), ou seja, novilhas de 14 ou 20 meses apresentaram a mesma taxa de prenhez.
Observando a taxa de prenhez dentre os protocolos de indução utilizados, não houve diferença entre eles $(P=0.869)$.

Tabela 4. Resultado do diagnóstico de gestação de acordo com o uso de protocolo de indução comparado ao grupo não induzido, de novilhas Nelore.

Table 4. Result of pregnancy diagnosis according to the use of induction protocol compared to the non-induced group of Nellore heifers.

\begin{tabular}{ccccc}
\hline & \multicolumn{4}{c}{ Prenhez } \\
\hline & Número de Novilhas & Positiva & Negativa & \\
\hline Grupos & $\mathrm{N}$ & $\mathrm{N}$ & $\mathrm{N}$ & p value \\
\hline 1 & 534 & 289 & 245 & 0.0068 \\
2 & 539 & 289 & 250 & 0.0107 \\
3 & 455 & 207 & 248 & - \\
\hline Total & 1528 & 785 & 743 & - \\
\hline
\end{tabular}

*Grupo 1: P4 injetável; Grupo 2: implante de progesterona + cipionato de estradiol; Grupo 3: não receberam protocolo de indução.

Tabela 5: Resultado do diagnóstico de gestação de acordo com o uso de protocolo de indução relacionado com a idade de novilhas Nelore.

Table 5. Result of the pregnancy diagnosis according to the use of an induction protocol related to the age of Nellore heifers.

\begin{tabular}{cccccc}
\hline & & \multicolumn{4}{c}{ Prenhez } \\
\hline & $\begin{array}{l}\text { Número de } \\
\text { Novilhas }\end{array}$ & Idade & Positivo & Negativo & \\
\hline Grupos & $\mathrm{N}$ & & $\mathrm{N}$ & $\mathrm{N}$ & p value \\
\hline \multirow{2}{*}{1} & \multirow{2}{*}{534} & 14 meses & 118 & 113 & \multirow{2}{*}{0.2187} \\
& \multirow{2}{*}{2} & 20 meses & 171 & 132 & \\
\hline Total & \multirow{2}{*}{539} & 14 meses & 121 & 120 & \multirow{2}{*}{0.1534} \\
\hline
\end{tabular}

*Grupo 1: P4 injetável; Grupo 2: implante de progesterona + cipionato de estradiol.

\section{DISCUSSÃO}

Todas as novilhas avaliadas apresentaram ECC entre 3 e 3,5. Rosa et al. (2011) afirmaram que o ECC acima de 2,5 é o indicado na seleção da matriz, pois este escore proporciona a fêmea desmamar boas crias e facilidade de manutenção da condição corporal ou para se recuperar de forma fácil no período pós-parto até a desmama.

Pesquisa realizada por White et al. (2016), demonstrou que novilhas que possuíam maior ECC ( $>5$, escala de 1 a 9) apresentaram taxa de prenhez de $58,9 \%$, enquanto às novilhas com menor escore de condição corporal $(<5)$ apresentaram 50\%. Estes dados demonstram que as novilhas que compunham o grupo que participou deste estudo apresentaram excelente ECC, o que as classificou como aptas para participar da estação reprodutiva.

Todas as novilhas estavam com peso acima de $270 \mathrm{~kg}$. Geralmente novilhas púberes são mais pesadas ao desmame que novilhas não púberes, por isso apresentam puberdade mais precoce (MONTEIRO et al., 2013). Endecott et al. (2013) afirmaram que o melhor retorno econômico da atividade ocorre quando a fêmea que irá iniciar a atividade reprodutiva esteja com 50 a $57 \%$ do peso adulto.

Um fato importante a salientar é que a avaliação do peso de forma isolada pode não apresentar estimativa correta de idade a puberdade e sucesso reprodutivo da fêmea, isso porque os animais apresentam diferenças fenotípicas (altura, comprimento, profundidade), além de que o peso tem baixa correlação com deposição de gordura subcutânea (AYRES et al., 2009; KOURY FILHO et al., 2009). 
Nesta pesquisa os animais além de apresentar peso adequado, também possuíam ECC apropriado, características estas que reforçam que as novilhas que foram avaliadas estavam hábeis para ingressar na estação reprodutiva.

Os animais induzidos apresentaram melhores taxa de prenhez quando comparados aos animais não induzidos. Em estudo realizado por Araujo et al. (2019), os resultados demonstraram que as novilhas pré-puberes induzidas tiveram taxa de ovulação de 82,22\%. Assim como Cadima (2018), que afirmou em seu estudo que a taxa de resposta ao protocolo de indução foi de $73,9 \%$. Este fato pode explicar a melhor taxa de prenhez de novilhas pré-puberes induzidas quando comparadas a não induzidas desta pesquisa.

As novilhas de 14 ou 20 meses apresentaram a mesma taxa de prenhez. Animais Bos taurus indicus, como os utilizados neste estudo, são animais mais tardios e costumam iniciar a ciclicidade em torno dos 22 a 36 meses, o que faz com que a idade ao primeiro parto ocorra entre o terceiro ou quarto ano de vida nos rebanhos brasileiros (ROMANO et al., 2007; MALHADO et al., 2013). Desta forma a grande vantagem em instituir precocemente o protocolo de indução em novilhas com cerca de 14 meses de idade é diminuir a idade ao primeiro parto e aumentar assim a produtividade da matriz.

Rebanhos cujas matrizes tem o primeiro parto aos 24 meses, apresentam maior produtividade e retorno econômico quando comparado a rebanhos cuja idade ao primeiro parto é aos 36 ou 48 meses de idade (ARAUJO et al., 2018). Por isso, sugere-se que seja realizada a indução de ciclicidade em novilhas com cerca de 14 meses de idade pois estas respondem ao protocolo positivamente da mesma forma que novilhas aos 20 meses. Vale ressaltar que, a nutrição, genética e sanidade são pontos importantes para atingir o objetivo de melhoria nos índices reprodutivos destas novilhas em idade precoce.

O protocolo utilizando apenas P4 injetável apresentou os mesmos resultados do tratamento que utilizou dispositivo intravaginal reutilizável associado ao cipionato de estradiol, dados estes semelhantes aos encontrados por Leme (2017).

Desta forma, como alternativa ao protocolo de indução de puberdade em novilhas que utiliza dispositivos intravaginais, o uso de P4 injetável pode ser uma ferramenta bastante interessante, pois é um protocolo que apresenta facilidade de manejo, além de evitar problemas sanitários devido a reutilização dos dispositivos intravaginais.

\section{CONCLUSÕES}

A progesterona é eficaz para indução de puberdade em novilhas Nelore a partir dos 14 meses de idade com peso médio de $270 \mathrm{~kg} \pm 9$.

Novilhas Nelore pré-puberes que passam por protocolos de indução hormonal apresentam melhores taxa de concepção logo na primeira IATF, quando comparadas a novilhas que não foram induzidas, independentemente da idade. Por isso, novilhas pré-puberes que estiverem com ECC e peso adequados devem ser induzidas e participar da estação reprodutiva o mais cedo possível visando a melhoria da produtividade e retorno econômico da atividade.

A utilização da P4 injetável pode ser uma alternativa eficaz para uso como protocolo de indução de novilhas Nelore pré-puberes, pois apresenta menor custo e mais praticidade de manejo quando comparada aos protocolos com uso de dispositivos intravaginais reutilizáveis.

\section{REFERÊNCIAS}

ARAUJO, A. C. C.; NONATO, M. S.; BEZERRA, A. R. A.; MURTA, D. C. R. X.; MURTA, D. V. F.; SANTOS, J. M. L.; SOUZA, R. B.; CARNEIRO, J. A. M. Induction of ovulation in heifers with memorandum of cyclicality. Brazilian Journal of Development, v. 5, n. 11, p. 24286-24290, 2019.

ARAUJO, A. C. R.; SALES, A. F. F.; FERREIRA, J. P. V.; NEVES NETO, J. T. Indução à puberdade em novilhas. In: Colóquio Estadual de Pesquisa Interdisciplinar e Congresso Nacional de Pesquisa Multidisciplinar, II-I. Anais... 2018.

AYRES, H.; FERREIRA, R. M.; SOUZA TORRESJÚNIOR, J. R. DE; DEMÉTRIO, C. G. B.; LIMA, C. G. DE; BARUSELLI, P. S. Validation of body condition score as a predictor of subcutaneous fat in Nelore (Bos indicus) cows. Livestock Science, v. 123, n. 2-3, p. 175179, 2009.2 DOI: https://doi.org/10.1016/j.livsci.2008.11.004

BÓ, G. A. Programs for fixed-time artificial insemination in South American beef cattle. Animal Reproduction Science, v. 15, n. 1, p. 952-962, 2018. DOI: http:/ /dx.doi.org/10.21451/1984-3143-AR2018-0025

CADIMA, G. P. Efeito da indução de puberdade em novilhas nelore no desempenho reprodutivo na estação de monta. 2018. 21f. Monografia (Graduação em Medicina Veterinária). Uberlândia: Universidade Federal de Uberlândia, 2018.

CARDOSO, D.; NOGUEIRA, G. P. Mecanismos neuroendócrinos envolvidos na puberdade de novilhas. Arquivo Ciência Veterinária Zoologia, v. 10, n. 1, p. 59-67, 2007.

DAY, M. L.; NOGUEIRA, G. P. Management of age at puberty in beef heifers to optimize efficiency of beef production. Animal Frontiers, v. 3, n. 4, p. 6-11, 2013. DOI: https://doi.org/10.2527/af.2013-0027

ELER, J. P.; FERRAZ, J. B. S.; TEIXEIRA, L. A. Seleção para precocidade sexual em novilhas de corte. In: PIRES, A. V. (Ed.). Bovinocultura de corte. Piracicaba: Fealq, 2010. p. 801-811.

ENDECOTT, R. L.; FUNSTON, R. N.; MULLINIKS, J. T.; ROBERTS, A. J. Implications of beef heifer development systems and lifetime productivity. Journal of Animal Science, v. 91, p. 1329-1335, 2013.

FERREIRA, E. M.; FERRAZ JUNIOR, M. V. C.; NEPOMUCENO, D. D.; BIEHL, M. V.; Mendes, C.Q.; PIRES, A. V. Efeito da produção de novilhas à pasto sobre a puberdade. In: Rosane Cláudia Rodrigues; Michelle de Oliveira Parante. (Org.). SIMPRUPASTO: $O$ uso da ciência e de tecnologias para a mudança de paradigmas. 1ed. Chapadinha: Edufma, 2012, v. 1, p. 128- 158

FIGUEIREDO, D. M.; PAULINO, M. F.; DETMANN, E.; SOUZA, M. G.; COUTO, V. R. M.; SALES, F. L. Estratégias de suplementação para antecipação da idade à puberdade para novilhas de corte em pastagem tropical. Acta Scientiarum, Animal Sciences, Maringá, v. 30, n .4, p. 415-423, 2008. 
HAFEZ, E. S.; HAFEZ, B. Fisiologia da reprodução. 7 ed. São Paulo: Manole, 2004. 513p.

IBGE_INSTITUTO BRASILEIRO DE GEOGRAFIA E ESTATÍSTICA. Pesquisa da pecuária municipal. $2018 . \quad$ Disponível em: https://sidra.ibge.gov.br/pesquisa/ppm/quadros. Acesso em: 28 jul. 2020.

KOURY FILHO, W.; ALBUQUERQUE, L. G. de; ALENCAR, M. M. de; FORNI, S.; SILVA, J. A. I. de V.; LÔBO, R. B. Estimativas de herdabilidade e correlações para escores visuais, peso e altura ao sobreano em rebanhos da raça Nelore. Revista Brasileira de Zootecnia, Viçosa, v. 38, n. 12, p. 2362-2367, 2009. DOI: $\quad$ https://doi.org/10.1590/S151635982009001200010.

LEMES, K. M. Comparação da eficiência de diferentes formulações à base de progesterona para indução da puberdade e desempenho reprodutivo em novilhas da raça Nelore. 2017. 80f. Tese (Doutorado em Reprodução Animal). Pirassununga: Universidade de São Paulo, 2017.

MALHADO, C. H. M.; MALHADO, A. C. M.; MARTINS FILHO, R.; CARNEIRO, P. L. S.; PALA, A.; ADRIÁN CARRILLO, J. Age at first calving of Nellore cattle in the semi-arid region of northeastern Brazil using linear, threshold, censored and penalty models. Livestock Science, v. 154, n. 1-3, p. 28-33, 2013. DOI: https://doi.org/10.1016/j.livsci.2013.02.021

MARSON, E. P.; GUIMARAES, J. D.; MIRANDA NETO, T. Puberty and sexual maturity in beef heifers. Revista Brasileira de Reprodução Animal, v. 28, n. 1, p. 3-12, 2004.

MONTEIRO, F. M.; MERCADANTE, M. E. Z.; BARROS, C. M.; SATRAPA, R. A.; SILVA, J. A. V; OLIVEIRA, L. Z.; SARAIVA, N. Z.; OLIVEIRA, C. S.; GARCIA, J. M. Reproductive tract development and puberty in two lines of Nellore heifers selected for postweaning weight. Theriogenology, v. 80 , n. 1, p. 10-17, 2013. DOI: https://doi.org/10.1016/j.theriogenology.2013.02.013

PERRY, G. A. Factors affecting puberty in replacement beef heifers. Theriogenology, v. 86, p. 373-378, 2016. DOI: https://doi.org/10.1016/j.theriogenology.2016.04.051
QUADROS, S. A. F. de; LOBATO, J. F. P. Bioestimulação e comportamento reprodutivo de novilhas de corte. Revista Brasileira de Zootecnia, Viçosa, v. 33, n. 3, p. 679-683, 2004. DOI: https://doi.org/10.1590/S151635982004000300016

ROMANO, M. A.; BARNABE, V. H.; KASTELIC, J. P.; OLIVEIRA, C. A. de; ROMANO, R. M. Follicular dynamics in heifers during pre-pubertal and pubertal period kept under two levels of dietary energy intake. Reproduction in Domestic Animals, v. 42, n. 6, p. 616-622, 2007.

ROSA, A. N.; SILVA, L. O. C.; THIAGO, L. R. L. S. Avaliação do escore da condição corporal em zebuínos. 2011. Disponível em < http://geneplus.cnpgc.embrapa.br/upload/artigos/Con dicaoCorporalZebuinos20052016.pdf>. Acesso em 28 jul. 2020.

SANTOS, M. H.; FERRAZ JÚNIOR, M. V. C.; POLIZEL, D. M.; BARROSO, J. P. R.; MISZURA, A. A.; MARTINS, A. S.; PIRES, A. V. Decreasing from 9 to 7 days the permanence of progesterone inserts make possible their use up to 5 folds in suckled Nellore cows. Theriogenology, v. 111, p. 56-61, 2018. DOI: 10.1016/j.theriogenology.2018.01.017

SOUZA, R. T.; GONÇALVES, J. L.; SANTOS, S. F.; FERNANDES, A. M. F.; RICCI, G. D. Fatores Relacionados ao Desenvolvimento Reprodutivo em Novilhas Nelore: Revisão. PUBVET, v.12, p.1-10, 2018.

VASCONCELOS, J. L. M. Reproductive programs for beef cattle: incorporating management and reproductive techniques for better fertility. Animal Reproduction Science, v.14, n.3, p.547-557, 2017. DOI: 10.21451/1984-3143-AR998

WHITE, S. S.; KASIMANICKAM, R. K.; KASIMANICKAM, V. R. Fertility after two doses of PGF $2 \alpha$ concurrently or at 6-hour interval on the day of CIDR removal in 5-day CO-Synch progesterone-based synchronization protocols in beef heifers. Theriogenology, v. 86, n. 3, p. 785-790, 2016. DOI: https://doi.org/10.1016/j.theriogenology.2016.02.032 